\title{
Assessing Arterial Stiffness using the Oscillometric Method in Patients with Diabetes and/or Hypertension \\ Correia $D^{1,2^{*}}$, Resende LAPR ${ }^{1}$, Resende JAM ${ }^{1}$, Silva MAV ${ }^{1}$, Resende EAMR ${ }^{1}$ and Silva VJD ${ }^{1}$ \\ ${ }^{1}$ University Hospital of the Federal University of Triangulo Mineiro, Uberaba, Minas Gerais, Brazil \\ ${ }^{2}$ Department of Tropical Medicine and Infectology, Federal University of the Triangulo Mineiro, Uberaba, Minas Gerais, Brazil
}

"Corresponding author: Correia D, Department of Tropical Medicine and Infectiology, Federal University of the Triangulo Mineiro, Uberaba, Minas Gerais, Brazil, Tel: +5534 3318-5254; E-mail: dalmo@mednet.com.br

Received: September 18, 2018; Accepted: October 10, 2018; Published: October 15, 2018

Copyright: @ 2018 Correia D, et al. This is an open-access article distributed under the terms of the creative commons attribution license, which permits unrestricted use, distribution, and reproduction in any medium, provided the original author and source are credited.

\section{Abstract}

Introduction: The parameters of pulse wave analysis, an emerging modality, are strong predictors of cardiovascular events and all-cause mortality. Systemic arterial hypertension and type 2 diabetes mellitus promote arterial stiffness, thereby modifying the characteristics of the pulse wave. This study analyzed and compared the pulse wave parameters of patients with diabetes and/or hypertension, using the oscillometric method.

Methods: In this cross-sectional study, the 110 study subjects were divided into three groups: Hypertensivediabetic, hypertensive, and diabetic. An oscillometric pulse wave test was performed using Mobil-O-Graph ${ }^{\circ}$ PWA equipment. The following variables were assessed: Age, body mass index, augmentation index, pulse wave velocity, peripheral arterial pressure, and central arterial pressure.

Results: The mean age of the study population was 61.3 years (standard deviation: 1.2). The mean body mass index was $29.3 \mathrm{~kg} / \mathrm{m}^{2}$ (standard deviation: 2.9). The hypertensive-diabetic group had the highest mean pulse wave velocity and peripheral and central arterial pressure values. Moreover, the systolic peripheral and central arterial pressures of the hypertensive-diabetic group were significantly higher than those of the other two groups $(p<0.05)$. All analyzed parameters were strongly correlated between the diabetic group and the hypertensive group $(p<0.0001)$

Conclusion: Patients with overlapping cardiovascular risk factors of diabetes and hypertension had the highest PWV and peripheral and central arterial pressure values. Hypertension and diabetes, as isolated conditions, were equivalent in their alterations of pulse wave analysis parameters.

Keywords: Pulse wave analysis; Arterial stiffness; Hypertension; Diabetes

\section{Introduction}

Globally, cardiovascular diseases account for approximately 17.5 million (or $31 \%$ of all) deaths annually [1]. Arterial hypertension plays an important role as, out of 19 risk factors, it is the main cause of cardiovascular mortality. Annually, hypertension is directly responsible for an estimated 7.6 million premature deaths [2,3]. Also, of concern is diabetes mellitus (DM). The International Diabetes Federation estimated that in 2014, approximately 387 million adults were affected by DM worldwide. In addition, there is a strong association between DM and macro- and microvascular complications, with an emphasis on cardiovascular disease $[4,5]$. Considering the burden of these two conditions, reliable markers of cardiovascular risk are essential in medical practice.

Among the new methods available to evaluate cardiovascular disease risk, assessment of arterial stiffness via pulse wave analysis (PWA) is increasingly being recognized as a promising approach. Increased arterial stiffness implies augmented pulse wave velocity (PWV) throughout the large arteries and premature return from reflected peripheral waves to the ascending aorta and left ventricle; this leads to increased cardiac afterload, increased myocardial oxygen consumption, and left ventricular hypertrophy [6-13]. Hence, arterial PWA is an important means of assessing arterial stiffness and cardiovascular risk. Elevations in pulse pressure, PWV, and the augmentation index are associated with established risk factors for cardiovascular diseases, including DM, hypertension, older age, obesity, and dyslipidemia [14-20].

Arterial stiffness can be measured using various methods. The gold standard is invasive intra-aortic catheter measurement. However, a new oscillometric method that is both reproducible and non-invasive, has recently been developed. This method allows measurement of peripheral and central blood pressure and PWA parameters, specifically PWV, by transforming the brachial pulse waveform [21]. This new approach can be applied in the outpatient setting and constitutes a simple and useful tool for primary care units. Validation studies suggest that the measurements obtained using this new method are strongly correlated with those of the gold standard method [22-24].

In this study, we performed PWA in individual with isolated hypertension, isolated DM, and concomitant hypertension and DM. Our aim was to evaluate the importance of DM and hypertensionalone and in combination-on arterial pulse wave characteristics and to compare groups of patients with these established cardiovascular risk factors, so as to determine the usefulness of the oscillometric method 
in identifying early pathologic changes in individuals affected by these extremely prevalent conditions.

\section{Materials and Methods}

\section{Study population}

This study analyzed the data of 110 individuals managed at the academic hypertension and diabetes outpatient service of the Federal University of Triangulo Mineiro, located in Uberaba, Minas Gerais State, Brazil between June 2016 and May 2017. These individuals were divided into three groups: Hypertensive (40 patients), hypertensivediabetic (40 patients), and diabetic (30 patients). Patients attending regular follow-up at the outpatient service for management of hypertension and/or DM were included. The exclusion criteria were age $<18$ years, underlying conditions requiring recent hospitalization, heart failure (functional class III or IV of the New York Heart Association), and end-stage kidney disease. All individuals underwent clinical examination.

The study protocol followed the principles of the Declaration of Helsinki and was approved by the institutional ethics committee (protocol number CAAE 57300816.6.0000.5154). Written informed consent was obtained from all participants.

\section{Clinical data}

All individuals answered questions pertaining to personal and clinical data. Patients underwent conventional sphygmomanometer arterial blood pressure measurement according to the Brazilian Hypertension Society guidelines; the diagnosis of hypertension was based on these guidelines [25]. DM was diagnosed according to the recommendations of the American Diabetes Association [26]. We did not select specific subgroup of diabetic patients.

\section{Pulse wave analysis}

In an appropriate room and after a rest period of at least $30 \mathrm{~min}$, we performed PWA using the oscillometric method; the device used also measures peripheral and central blood pressures. The device-the Mobil-O-Graph ${ }^{\oplus}$ PWA-EMI GmbH (Stolberg, Germany)-is a commercially available brachial oscillometric ambulatory blood pressure monitor that has been validated according to European Society of Hypertension recommendations [27,28]. To perform the measurements, a common cuff was centered on the left upper arm; cuff size was chosen according to the circumference of the mid upper arm. The generation of central aortic blood pressure curves from brachial pulse waves was based on a previously published algorithm that integrates arterial impedance and aortic hemodynamic into a mathematical model $[23,29]$. Four consecutive measurements were taken every $2 \mathrm{~min}$, in accordance with the general recommendations for clinical studies [11,30-32].

\section{Statistical analysis}

Data were entered Microsoft Excel software and were analyzed using GraphPad Prism software, version 7.00 for Windows (GraphPad Software, La Jolla California USA, www.graphpad.com). All results are expressed as the mean \pm standard deviation. Student's t-test and Wilcoxon's signed rank test were used to compare continuous variables between groups. Pearson correlation coefficients were calculated to evaluate the degree of association between variables. $p$-values $<0.05$ were considered indicative of statistical significance.

\section{Result}

The mean age of the study population was $61.3 \pm 1.2$ years and $42.7 \%$ of participants were men. The mean body mass index was $29.3 \pm$ 2.9 (Table 1).

\begin{tabular}{|l|l|l|l|}
\hline Characteristic & $\begin{array}{l}\text { With } \\
\text { diabetes } \\
(\mathbf{n = 3 0 )}\end{array}$ & $\begin{array}{l}\text { With } \\
\text { hypertension } \\
(\mathbf{n = 4 0 )}\end{array}$ & $\begin{array}{l}\text { With } \\
\text { hypertension } \\
\text { and diabetes } \\
(\mathbf{n = 4 0 )}\end{array}$ \\
\hline Female sex, $\mathrm{n}(\%)$ & $17(56.6)$ & $25(62.5)$ & $26(65)$ \\
\hline Age (years), mean $\pm \mathrm{SD}$ & $59.7 \pm 9.1$ & $60.1 \pm 9.8$ & $62 \pm 7.8$ \\
\hline $\begin{array}{l}\text { Body mass index }\left(\mathrm{kg} / \mathrm{m}^{2}\right) \\
\text { mean } \pm \mathrm{SD}\end{array}$ & $25.8 \pm 3.4$ & $30.9 \pm 5.45$ & $30.8 \pm 4.6$ \\
\hline
\end{tabular}

Table 1: Characteristics of the study population.

The hypertensive-diabetic group had the highest mean PWV and peripheral and central systolic arterial pressure values. There were significant differences in systolic peripheral and central pressures and pulse pressures between the hypertensive-diabetic group and the other two groups $(\mathrm{p}<0.05)($ Table 2).

\begin{tabular}{|c|c|c|c|}
\hline PWA parameter & $\begin{array}{l}\text { With } \\
\text { hypertension }\end{array}$ & $\begin{array}{l}\text { With } \\
\text { hypertension } \\
\text { and diabetes }\end{array}$ & With diabetes \\
\hline Age (years) & $60 \pm 12$ & $62 \pm 11$ & $62 \pm 11$ \\
\hline Body mass index $\left(\mathrm{kg} / \mathrm{m}^{2}\right)$ & $31.0 \pm 7.4$ & $31.0 \pm 5.8$ & $26.0 \pm 4.8$ \\
\hline Augmentation index & $29 \pm 13$ & $30 \pm 11$ & $30 \pm 11$ \\
\hline Reflection index & $67.0 \pm 7.0$ & $64.0 \pm 6.4$ & $65.0 \pm 5.3$ \\
\hline Pulse wave velocity & $8.9 \pm 2.2$ & $9.6 \pm 1.7$ & $9.3 \pm 1.6$ \\
\hline $\begin{array}{l}\text { Peripheral systolic } \\
\text { pressure }\end{array}$ & $139 \pm 20$ & $150 \pm 23$ & $138 \pm 13$ \\
\hline $\begin{array}{l}\text { Peripheral diastolic } \\
\text { pressure }\end{array}$ & $85 \pm 13$ & $90 \pm 17$ & $85 \pm 8.5$ \\
\hline Peripheral pulse pressure & $54 \pm 14$ & $60 \pm 25$ & $52 \pm 10$ \\
\hline Central systolic pressure & $129 \pm 19$ & $136 \pm 22$ & $127 \pm 11$ \\
\hline Central diastolic pressure & $87.0 \pm 13.0$ & $92.0 \pm 18.0$ & $87.0 \pm 8.8$ \\
\hline Central pulse pressure & $43.0 \pm 13.0$ & $44.0 \pm 12.0$ & $40.0 \pm 7.8$ \\
\hline \multicolumn{4}{|c|}{$\begin{array}{l}\text { Data are presented as the mean } \pm \text { standard deviation. } \\
\mathrm{p} \text { value for peripheral systolic pressure: } 0.003 \text {-with hypertension and diabetes } \\
\text { vs. with hypertension and with hypertension and diabetes } v s \text {. with diabetes. } \\
\mathrm{p} \text { value for central systolic pressure: } 0.0095 \text {-with hypertension and diabetes } v s \text {. } \\
\text { with diabetes and } 0.036 \text {-with hypertension and diabetes } v s \text {. with hypertension. } \\
\text { p value for pulse wave velocity: Non-significant for all groups. }\end{array}$} \\
\hline
\end{tabular}

Table 2: Pulse wave analysis (PWA) parameters.

Figure 1 illustrates the correlations in the analyzed parameters between the hypertensive and diabetic groups; strong correlations were observed $(\mathrm{p}<0.0001)$. 


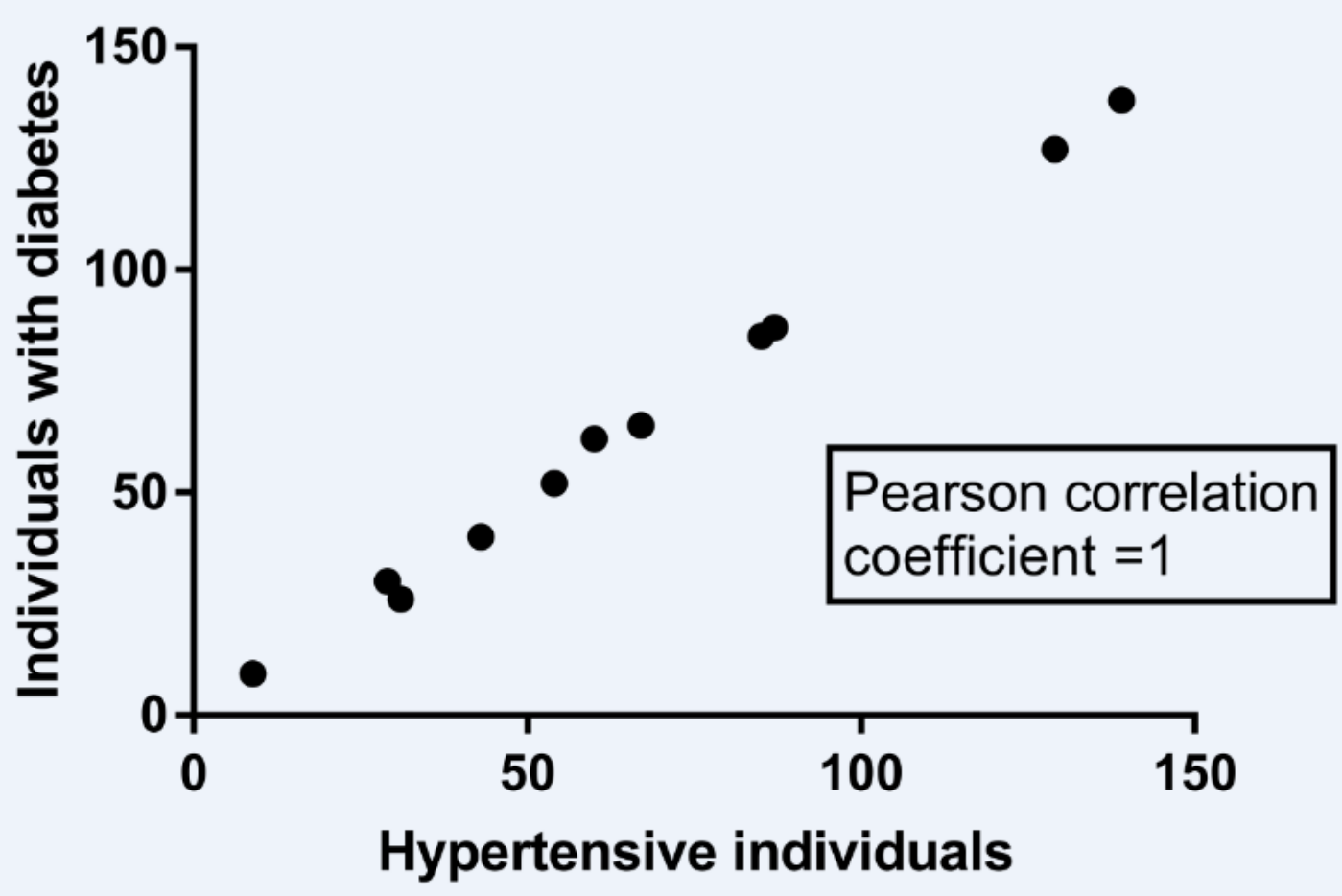

Figure 1: Correlations in the analyzed parameters between the hypertensive and diabetic groups.

\section{Discussion}

The results of this study illustrate the association between arterial stiffness assessed by PWA and two important diseases: hypertension and diabetes. These diseases are highly prevalent, and their pathophysiologic mechanisms compromise arterial properties. The early identification of vascular alterations, therefore, may be an extremely useful approach in the context of preventive medicine $[1,2,6,8]$. Large-scale diagnostic tools that are inexpensive and capable of identifying morbid conditions at an early stage, are crucial in providing quality healthcare. The oscillometric method of obtaining pulse wave parameters is of great value in this sense and has the benefit of being applicable in outpatient settings. The results of the present study are consistent with the current evidence on this topic $[19,20,22]$.

Our study presented an interesting statistical evaluation of the correlation of pulse wave parameters between the isolated hypertensive and isolated diabetic groups-strong correlations were observed for all parameters analyzed (age; peripheral, central and pulse pressure; PWV; and the augmentation index), with a Pearson coefficient of 1 . These data suggest that there is a degree of equivalence in the harmful vascular effects exerted by DM and hypertension. Many mechanisms could be involved in the process of arterial impairment, including systemic inflammation, local mechanical injury, and vessel remodelling; however, the mechanisms have not been fully elucidated, and more studies are needed on this topic $[2,10]$.

Another relevant aspect of our study was the evaluation of a group of patients with concomitant hypertension and DM. In the PWA, this group had the highest PWV, although the difference was not statistically significant; the peripheral and central systolic pressures were, though, significantly higher than those in the other two groups. These results are consistent with the findings of previous studies. These data illustrate the importance of assessing cardiovascular risk factors in individual's hypertension and/or DM.

Our study has some limitations. The study population was small and in addition, there was no distinction between patients in terms of the time from diagnosis of hypertension and/or DM. Future studies could assess the association between arterial PWA and evaluation by means of diagnostic tools already well established in medical practice, such as echocardiography, coronary computed tomography angiography, and glycosylated haemoglobin measurement. As well as the study of pulse wave parameters in subgroups of hypertensive and diabetic patients with declared target organ damage, especially at the renal level. Moreover, evaluation of arterial stiffness via pulse wave form components requires further research to support the routine use of this approach. Such studies may provide important evidence for the use of this cardiovascular risk marker.

\section{Conclusion}

Patients with overlapping cardiovascular risk factors (DM and hypertension) had the highest PWV and peripheral and central arterial pressure values. Hypertension and DM as isolated conditions were equivalent in altering the arterial PWA parameters.

\section{Interest conflicts}

There are no conflicts of interest for the present study. 


\section{Financial Support}

This work was supported by the National Council for Scientific and Technological Development (CNPq).

\section{References}

1. http://www.who.int/cardiovascular_diseases/en/.

2. Lawes CM, Vander Hoorn S, Rodgers A (2008) Global burden of bloodpressure-related disease, 2001. Lancet 371: 1513-1518.

3. Da Silva MA (2014) Diagnostic performance evaluation and agreement between multiple blood pressure measurements in the outpatient office with ambulatory measurements. Dissertation (Master degree): Technology Management and Innovation in Health, Syrian-Lebanese Institute of Education and Research, São Paulo p: 78.

4. Atlas D (2015) International Diabetes Federation. IDF Diabetes Atlas, $7^{\text {th }}$ edn. Brussels, Belgium: International Diabetes Federation.

5. Standards of Medical Care in Diabetes (2015) Summary of revisions. Diabetes Care 38: S4.

6. Pizzi O, Brandão AA, Magalhães ME, Pozzan R, Brandão AP (2006) Pulse wave-velocity method and its prognostic implications in hypertension. Rev Bras Hipertens 13: 59-62.

7. Safar ME, London BM (1994) The arterial system in human hypertension, in Swales JD (ed): Textbook of Hypertension. London, Blackwell Scientific Publications pp: 85-102.

8. O'rourke MF, Pauca A, Jiang XJ (2001) Pulse wave analysis. Br J Clin Pharmacol 51: 507-522.

9. Asmar R, O'Rourke M, Safar M (1999) Arterial stiffness and pulse wave velocity: Clinical applications ( $1^{\text {st }}$ ed.) Paris: Elsevier.

10. Zieman SJ, Melenovsky V, Kass DA (2005) Mechanisms, pathophysiology, and therapy of arterial stiffness. Arterioscler Thromb Vasc Biol 25: 932-943.

11. Laurent S, Cockcroft J, Van Bortel L, Boutouyrie P, Giannattasio C, et al. (2006) Expert consensus document on arterial stiffness: Methodological issues and clinical applications. Eur Heart J 27: 2588-2605.

12. Nichols WW (2005) Properties of the arterial wall: Theory. McDonald's blood flow in arteries. London: Arnold.

13. Brandão AA, Amodeo C, Alcântara C, Barbosa E, Nobre F, et al. (2017) I Luso-Brazilian positioning on central arterial pressure. Arq Bras Cardiol 108: 100-108.

14. Laurent S, Kingwell B, Bank A, Weber M, Struijker-Boudier H (2002) Clinical applications of arterial stiffness: Therapeutics and pharmacology. Am J Hypertens 15: 453-458.

15. Safar ME, London GM (2000) Therapeutic studies and arterial stiffness in hypertension: Recommendations of the European Society of Hypertension. J Hypertens 18: 1527-1535.

16. Zoungas S, Asmar RP (2007) Arterial stiffness and cardiovascular outcome. Clin Exp Pharmacol Physiol 34: 647-651.
17. Williams B, Lacy PS, Thom SM, Cruickshank K, Stanton A, et al. (2006) Differential impact of blood pressure-lowering drugs on central aortic pressure and clinical outcomes: Principal results of the Conduit Artery Function Evaluation (CAFE) study. Circulation 113: 1213-1225.

18. McEniery CM, Cockcroft JR (2007) Does arterial stiffness predict atherosclerotic coronary events? Adv Cardiol 44: 160-172.

19. Vlachopoulos C, Aznaouridis K, Stefanadis C (2010) Prediction of cardiovascular events and all-cause mortality with arterial stiffness: A systematic review and meta-analysis. J Am Coll Cardiol 55: 1318-1327.

20. Laurent S, Boutouyrie P, Asmar R, Gautier I, Laloux B, et al. (2001) Aortic stiffness is an independent predictor of all-cause and cardiovascular mortality in hypertensive patients. Hypertension 37: 1236-1241.

21. Feistritzer HJ, Reinstadler SJ, Klug G, Kremser C, Seidner B, et al. (2015) Comparison of an oscillometric method with cardiac magnetic resonance for the analysis of aortic pulse wave velocity. PLoS one 10: e0116862.

22. Wassertheurer S, Kropf J, Weber T, Van Der Giet M, Baulmann J, et al. (2010) A new oscillometric method for pulse wave analysis: Comparison with a common tonometric method. J Hum Hypertens 24: 498-504.

23. Wassertheurer S, Mayer C, Breitenecker F (2008) Modeling arterial and left ventricular coupling for non-invasive measurements. Simul Model Pract Th 16: 988-997.

24. Hametner B, Wassertheurer S, Kropf J, Mayer C, Eber B, et al. (2013) Oscillometric estimation of aortic pulse wave velocity: Comparison with intra-aortic catheter measurements. Blood Press Monit 18: 173-176.

25. VI Brazilian Guidelines on Hypertension (2010) Brazilian Society of Cardiology/Brazilian Society of Hypertension/Brazilian Society of Nephrology. Arq Bras Cardiol 95: 1-51.

26. American Diabetes Association (2015) Classification and diagnosis of diabetes. Diabetes care 38: S8-S16.

27. Franssen PM, Imholz BP (2010) Evaluation of the Mobil-O-Graph new generation ABPM device using the ESH criteria. Blood Press Monit 15: 229-231.

28. Nunan D, Fleming S, Hametner B, Wassertheurer S (2014) Performance of pulse wave velocity measured using a brachial cuff in a community setting. Blood Press Monit 19: 315-319.

29. Weber T, Wassertheurer S, Rammer M, Maurer E, Hametner B, et al. (2011) Validation of a brachial cuff-based method for estimating central systolic blood pressure. Hypertension 58: 825-832.

30. Mancia G, De Backer G, Dominiczak A, Cifkova R, Fagard R, et al. (2007) Guidelines for the management of arterial hypertension: The task force for the management of arterial hypertension of the European Society of Hypertension (ESH) and of the European Society of Cardiology (ESC). J Hypertens 25: 1105-1187.

31. Van Bortel LM, Duprez D, Starmans-Kool MJ, Safar ME, Giannattasio C, et al. (2002) Clinical applications of arterial stiffness, Task Force III: Recommendations for user procedures. Am J Hypertens 15: 445-452.

32. Smulyan H, Lieber A, Safar ME (2015) Hypertension, diabetes type II, and their association: Role of arterial stiffness. Am J Hypertens 29: 5-13. 